\title{
Nucleotide variation at the no-on-transient $A$ gene in Drosophila littoralis
}

\author{
S Huttunen ${ }^{1}$, S Campesan ${ }^{2}$ and A Hoikkala ${ }^{1}$ \\ ${ }^{1}$ Department of Biology, University of Oulu, PO Box 3000, FIN-90014, Oulu, Finland; ${ }^{2}$ Department of Genetics, University of \\ Leicester, Leicester LE1 7RH, UK
}

\begin{abstract}
The no-on-transient $A$ (nonA) gene encodes a putative RNAbinding protein, and mutations in this gene are known to affect vision, male courtship song and viability in Drosophila melanogaster. Here we have sequenced the coding region of the nonA gene of Drosophila littoralis and compared it with those of Drosophila virilis and D. melanogaster. All portions of nonA appeared to be conserved between $D$. littoralis and $D$. virilis, while the $5^{\prime}$ region of the gene of these two species showed high divergence from that of a more distantly-related species, $D$. melanogaster. The same was true for the glycine
\end{abstract}

Keywords: nonA; D. littoralis; $D$. virilis; nucleotide variation

\section{Introduction}

The no-on-transient $A($ non $A)$ gene is one of the genes whose mutant alleles have been found to affect behaviour in Drosophila flies (see review by Yamamoto et al, 1997). The gene was cloned and sequenced in Drosophila melanogaster in 1990 simultaneously by two groups: Besser et al (1990) and Jones and Rubin (1990). The extensive sequence homology between the central domain of NONA and of the human proteins PSF (PTB-associated splicing factor; Patton et al, 1993), HeLa protein p54 ${ }^{\mathrm{nrb}}$ (nuclear RNA-binding protein; Dong et al, 1993) and the product of nonO (an octamer-binding protein; Yang et al, 1993) strongly suggests conservation of function over a period of divergence between human and fruit flies. Consequently, Dong et al (1993) named the phylogenetically conserved central domain of the above-mentioned genes a DBHS domain (Drosophila behaviour and human splicing).

non A-mutations were first isolated in screens for defects in phototactic behaviour in D. melanogaster (Hotta and Benzer, 1970), the most severe effects of mutations being optomotor blindness and the lack of transient spikes in the electroretinogram. Kulkarni et al (1988) also found some mutants to have disturbances in male courtship song, increasing the amplitude and polycyclicity of the sound pulses as a train of pulses proceeds. Rendahl et al (1996) found this mutation, called dissonance, to be

Correspondence: S Huttunen, Department of Biology, University of Oulu, PO Box 3000, FIN-90014, Oulu, Finland.

E-mail:susanna.huttunen@oulu.fi

Received 5 February 2001; accepted 6 August 2001 repeat regions. No significant deviation from neutrality was observed in the analysis of intraspecific nucleotide variation in $5^{\prime}$ or $3^{\prime}$ region of the non $A$ gene in $D$. littoralis population. Also, comparison of $D$. littoralis sequences with homologous sequence of $D$. virilis suggests that the gene is evolving neutrally in $D$. virilis group. Divergence of the $5^{\prime}$ regions between $D$. virilis group species and $D$. melanogaster could be a result of positive selection, but this finding is obscured by the long divergence time of the species groups.

Heredity (2002) 88, 39-45. DOI: 10.1038/sj/hdy/6800006

an allele of nonA (nonA $A^{\text {diss }}$ ) and showed it to be caused by an amino acid replacement (from Arg to Cys) at site 548 in nonA of D. melanogaster. Rendahl et al (1996) also demonstrated that novel point mutations introduced into the first RNA recognition motif of nonA cause disturbances in visual behaviour, male courtship song and the viability of the flies.

The non $A$ gene of $D$. virilis has been sequenced by Campesan et al (2001). They showed that the non $A$ genes of $D$. virilis and $D$. melanogaster are diverged at the $5^{\prime}$ region, but that the genes are highly conserved at the central and $3^{\prime}$ region. Species divergence in $5^{\prime}$ region could reflect lowered functional constraint, or it might be a consequence of positive selection affecting this gene region. To find out whether this region shows divergence also between more closely-related species, we have sequenced the coding region of the non $A$ gene of another $D$. virilis group species, $D$. littoralis, and compared it with that of $D$. virilis and $D$. melanogaster. We have also tested the neutrality of sequence evolution in $5^{\prime}$ and $3^{\prime}$ regions of the non $A$ gene by studying intraspecific nucleotide variation on these regions in $D$. littoralis and by comparing $D$. littoralis sequences with the $D$. virilis and $D$. melanogaster sequences. Additional interest in this work is due to the recent finding that non $A$ gene is located at the proximal end of the $X$ chromosome, where the gene or genes causing major differences between the songs of $D$. virilis and $D$. littoralis are known to be located (Päällysaho et al, 2001). Thus it is a good candidate gene for causing species differences in male courtship song in the $D$. virilis group. 


\section{Materials and methods}

\section{DNA extraction and PCR}

Genomic DNA from D. littoralis strain $1007\left(47^{\circ} \mathrm{N} 8^{\circ} \mathrm{E}\right.$, Zürich, 1970) males was extracted using the standard protocol of the Puregene DNA Isolation Kit (Gentra Systems). An approximately $4.3 \mathrm{~kb}$ region from exon 2 to exon 5 of the nonA locus was PCR-amplified using oligonucleotide primers CACAACTTCAAGCGCAGGCC CAA (forward) and CTAAAAACGACGTCGTCCCCATG (reverse) designed from $D$. virilis non $A$ sequence (GenBank accession number AJ298998). PCR was performed in $50 \mu \mathrm{l}$ reactions using two units of DynazymeEXT DNA polymerase (Finnzymes), $0.5 \mu \mathrm{M}$ of primers (Pharmacia), $360 \mu \mathrm{M}$ of each dNTP and $2 \mathrm{mM}$ of $\mathrm{MgCl}_{2}$ (Finnzymes). The PCR profile was the following: first denaturation at $94^{\circ} \mathrm{C}$ for $2 \mathrm{~min}$, then denaturation at $94^{\circ} \mathrm{C}$ for $30 \mathrm{~s}$, annealing and extension at $68^{\circ} \mathrm{C}$ for $3 \mathrm{~min}$ and $30 \mathrm{~s}$ repeated for 30 times, and a final synthesis at $68^{\circ} \mathrm{C}$ for $10 \mathrm{~min}$. The sequence for the $5^{\prime}$ region of the gene was first analysed through cDNA synthesis and then PCR amplified also from genomic DNA (see below; Nterminal region in exon 1). The PCR products were cloned using the TA Cloning Kit (Invitrogen) according to instructions of the supplier.

Genomic DNA was also extracted from a single male of D. virilis laboratory strain $1431\left(53^{\circ} \mathrm{N} 1^{\circ} \mathrm{W}\right.$, England, 1982) and from six wild-caught $D$. littoralis males from three sites in Finland (Sa: Savonlinna, $62^{\circ} \mathrm{N}, 29^{\circ} \mathrm{E}, \mathrm{Ku}$ : Kuopio, $63^{\circ} \mathrm{N} 27^{\circ} \mathrm{E}$ and Ou: Oulu, $65^{\circ} \mathrm{N} 25^{\circ} \mathrm{E}$ ). The DNA was PCR-amplified using oligonucleotide primers GTTTCTGTACGGAGCTGGA CGGTTG (forward) and GCCGCCACGATTGCGGTTG (reverse) for a $405 \mathrm{bp}$ sequence in exon 1 (N-terminal), primers ACAACA GATGCACC AAAAGCG (forward) and ACTCCTCATC GGTAATGTCATTGG (reverse) for a 514 bp sequence in exon 2 (N-terminal/Central domain), and primers CGCGAATCTGATAATGAGCG (forward) and CTGTC GCTGGTTATTTGCAC (reverse) for a $495 \mathrm{bp}$ sequence in exons 3 and 4 and intron 3 (C-terminal; see Figure 1). For PCR amplification the reaction volume was $50 \mu \mathrm{l}$, consisting of $\sim 100 \mathrm{ng}$ of genomic DNA, $1 \mu \mathrm{M}$ of each primer (Pharmacia), $200 \mu \mathrm{M}$ of each dNTP (Finnzymes), $1.5 \mathrm{mM}$ of $\mathrm{MgCl}_{2}$ (Finnzymes), $1 \times$ standard reaction buffer (Finnzymes) and $2 \mathrm{U}$ of DyNAzyme DNA polymerase (Finnzymes). The amplification profile was $3 \mathrm{~min}$ at $94^{\circ} \mathrm{C}$, followed by 35 cycles of $30 \mathrm{~s}$ at $94^{\circ} \mathrm{C}, 30 \mathrm{~s}$ at $58-64^{\circ} \mathrm{C}$, $30 \mathrm{~s}$ at $72^{\circ} \mathrm{C}$, and finally one cycle of $10 \mathrm{~min}$ at $72^{\circ} \mathrm{C}$. The annealing temperatures for the studied regions were $64^{\circ} \mathrm{C}, 58^{\circ} \mathrm{C}$ and $60^{\circ} \mathrm{C}$, respectively. The resulting fragment was purified from $1 \%$ agarose gel using the method of Glenn and Glenn (1994).

RNA isolation and reverse transcriptase PCR (RT-PCR) Isolation of the total RNA from adult D. littoralis 1007 males was performed according to manufacturer's instructions with TriPure ${ }^{\circledR}$ Isolation Reagent Kit (Boehringer Mannheim). To determine the exact exonintron boundaries without having to sequence the long intron sequences, various lengths of cDNAs of nonA were made using Enhanced Avian RT-PCR Kit (Sigma). The 5'RACE System for Rapid Amplification of cDNA Ends, Version 2.0 (GibcoBRL, Life Technologies) was used to isolate the remaining $5^{\prime}$ region of the $D$. littoralis non $A$ cDNA with a forward Abridged Anchor primer and reverse primers designed from the $D$. virilis sequence. The amplification was carried out using the following program: $94^{\circ} \mathrm{C} 2 \mathrm{~min} ; 35$ cycles of $94^{\circ} \mathrm{C} 30 \mathrm{~s}, 50^{\circ} \mathrm{C} 30 \mathrm{~s}$, $68^{\circ} \mathrm{C} 1 \mathrm{~min}$, and a final extension at $68^{\circ} \mathrm{C}$ for $10 \mathrm{~min}$.

\section{Sequencing}

A different sequencing strategy was used to obtain the first $D$. littoralis sequence (cloning) from that used for the population survey (direct sequencing of PCR products). All cloned sequences were confirmed from at least 10 independent clones to eliminate errors caused by misincorporation of nucleotides by DNA polymerase. Discrepancies arising were resolved also by direct sequencing of additional PCR products amplified from genomic DNA.

The purified PCR products from the two $5^{\prime}$ and one $3^{\prime}$ regions of the non $A$ gene from one $D$. virilis male (strain 1431) and wild-caught $D$. littoralis males were directly sequenced. Sequencing of both DNA strands was performed with an Applied Biosystems model 377 DNA sequencing system, using the ABI PRISM ${ }^{\circledR}$ BigDye $^{\mathrm{TM}}$ Terminator Cycle Sequencing Ready Reaction Kit (Perkin Elmer). Approximately $50 \mathrm{ng}$ of the PCR product was added to $4 \mu \mathrm{l}$ of dye terminator reaction mix and $6.4 \mathrm{pg}$ of sequencing primer (either forward or reverse used in initial PCR) with $2 \mu \mathrm{l}$ of $\times 5 \mathrm{TE}$ buffer in a final reaction volume of $20 \mu \mathrm{l}$.

\section{Computer analysis}

DNA sequences were edited using Chromas version 1.43 (http://trishul.sci.gu.edu.au/ conor/chromas.html) and the sequences were aligned using ClustalX (Thompson et al, 1997). The sequence analysis was made with Dnasis (Hitachi Software Engineering Co), Seqweb program

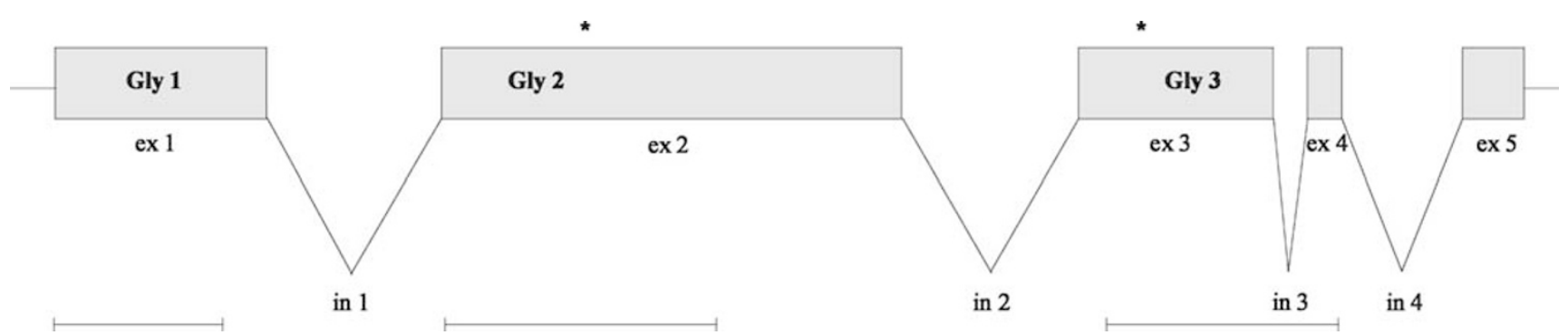

Figure 1 The schematic illustration of the nonA gene in D. littoralis. Introns are indicated by lines (not in scale) and exons by boxes. Gly1, Gly2 and Gly3 are glycine repeat regions. The black lines below the gene structure show the three regions sequenced from $18 \mathrm{D}$. littoralis males and one $D$. virilis male. The boundaries of amino terminal (N-term), central domain and carboxy terminal region (C-term) are marked by stars. 


$\frac{\mathrm{N} \text {-terminal }}{\mathrm{Gly} 1} \stackrel{\mathrm{Gly} 2}{ }$

11111111222222222333444444455555666677777778 256602333799000112234457135666722567001824577891 611219128425367365910842183258458510062191914283

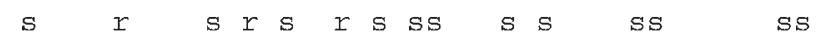

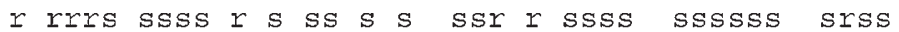
oul CAGCACCATCCTGGAGTTGTATTGGTTCGTTTCATCTAGGCCGGACGA

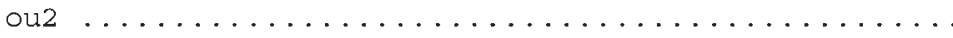

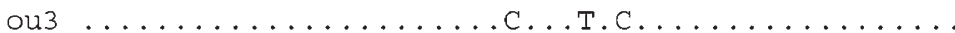
ou4 ................ . С...........

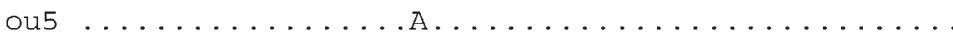

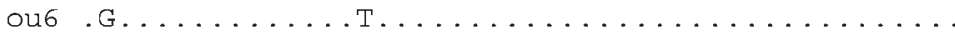
ku1 ................. . . ..........

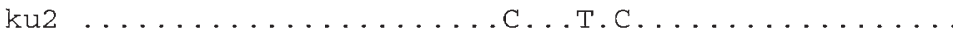

kuз ............... . . .........

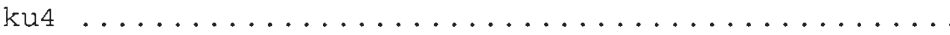

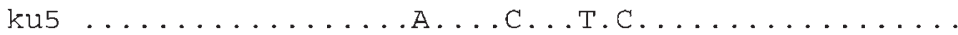

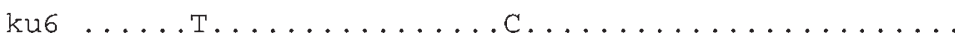

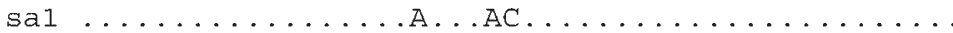

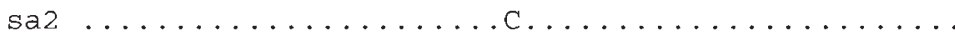

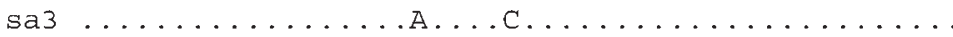
sa4 ...................АТ......

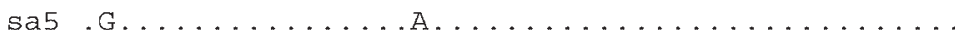

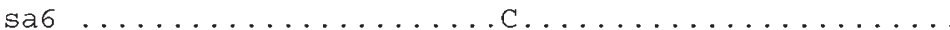
vir A. CGTA. CATT. T. TTCC.A-C.CACGTT. CCTT. . ACTATTA. CTCG

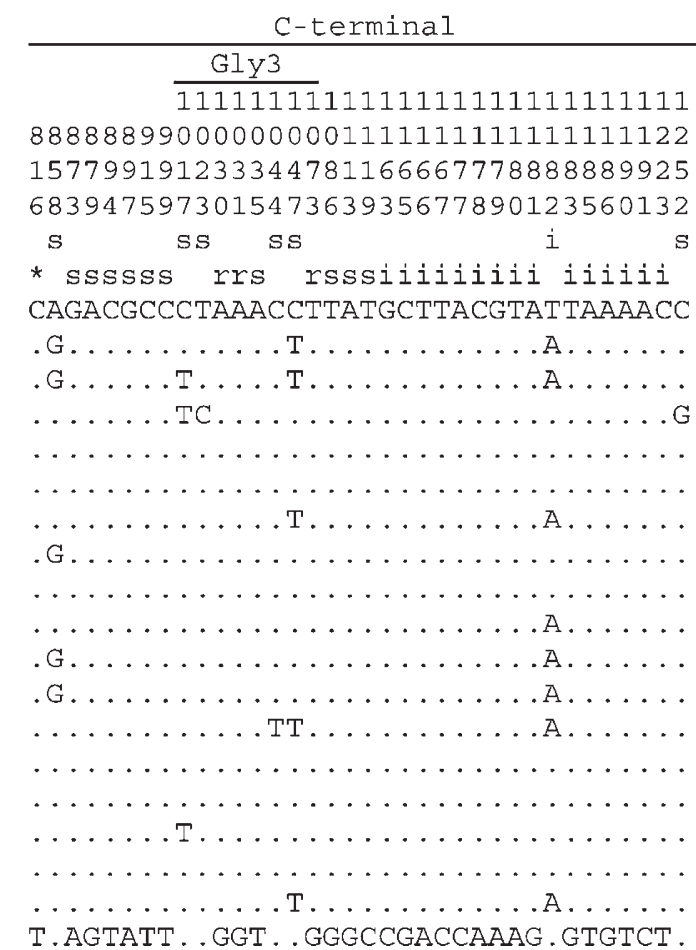

Figure 2 Variability in the analysed nonA gene regions in D. littoralis and D. virilis. Positions of the variable nucleotide sites are presented relative to the sequenced region. Dots represent the same nucleotide as in the first sequence. Abbreviations: $r=$ replacement substitution, $\mathrm{s}=$ synonymous substitution, $\mathrm{i}=$ intron site,${ }^{*}=$ triplet incomplete, $\mathrm{ou}=\mathrm{Oulu}, \mathrm{ku}=$ Kuopio, $\mathrm{sa}=$ Savonlinna and vir $=$ D. virilis $(\mathrm{strain} 1431$ ). Polymorphic sites among $D$. littoralis sequences are shown on the fourth lane and fixed differences between $D$. littoralis and $D$. virilis on the fifth lane.

version 10.0 of the GCG packet (http://seqweb.csc.fi/ gcg-bin/seqweb.cgi) and CodonW, version 1.4.2 (http: //bioweb.pasteur.fr/seqanal/interfaces/codonw.html). The estimates for the nucleotide variation were calculated using DnaSP version 3.5 (http://www.bio.ub.es/ julio/DnaSP.html), ProSeq (http://helios.bto.ed.ac.uk/ evolgen/filatov/proseq.html) and DNA Slider (http: //udel.edu/ mcdonald).

The coding region of the non $A$ gene from $D$. littoralis is deposited in GenBank (accession number AJ296178). The previously published non $A$ sequences from $D$. virilis (GenBank accession number AJ298998) and D. melanogaster (GenBank accession number M33496) were also used in this study. The 19 sequences listed in Figure 2 have been submitted to GenBank and have accession numbers AJ304304-369 for D. littoralis and AJ30470-72 for D. virilis.

\section{Results}

The coding region of the $D$. littoralis nonA gene The translated region of the non $A$ gene in $D$. littoralis is 2097 nucleotides long producing an open reading frame with homology to that of the $D$. virilis and $D$. melanogaster non $A$ genes. The structure of non $A$ gene (including the promotor region) has been described in $D$. melanogaster eg, by Besser et al (1990) and in D. virilis by Campesan et al (2001). The exon-intron organisation was the same in all three species (Figure 1), and the intron lengths were also almost identical between $D$. littoralis and $D$. virilis. The first and the second introns were about $2 \mathrm{kbp}$, the third intron $79 \mathrm{bp}$ and the fourth intron was $780 \mathrm{bp}$ long.

\section{Protein comparison}

Sequence analysis of $D$. littoralis nonA predicts a protein of 698 amino acids in comparison with 697 amino acids of $D$. virilis NONA and 700 amino acids of $D$. melanogaster NONA. As shown by Campesan et al (2001), the product of $D$. virilis non $A$ gene can be divided into conserved and non-conserved domains: N-terminal region, central domain and C-terminal region. The $\mathrm{N}$ - and C-terminals include stretches of repeated amino acids, the C-terminal also being rich in charged amino acids. The conserved central domain contains two tandemly repeated 80 amino acid RNA-binding domains (RRMs) in exon two, both of which contain RNP1 (eight amino acids) and RNP2 (six amino acids) motifs. These consensus sequences contain aromatic and basic residues, implicated in interactions between proteins and single-stranded nucleic acids (Kenan et al, 1991).

Excluding gaps, the overall amino acid identity between $D$. littoralis and D. virilis was $97.7 \%$ (97.8\% when conservative substitutions are included, Table 1). The respective percent identities between $D$. littoralis and $D$. melanogaster and between $D$. virilis and D. melanogaster were 77.8 and $77.6 \%$ (83.7 and $83.7 \%$ when conservative substitutions are included). A closer look at identities and similarities at different parts of the gene revealed that all portions of the gene are conserved between $D$. virilis and $D$. littoralis, while the N-termini of the NONA proteins in these two species are highly diverged from that of $D$. melanogaster. The central domain included only six amino acid substitutions between $D$. littoralis and $D$. virilis and 28 substitutions between $D$. littoralis and D. melanogaster. RNA-binding sites, which have been found to be highly 
Table 1 Comparison of nonA amino acid sequences of D. littoralis (lit), D. virilis (vir) and D. melanogaster (mel) overall and separately in amino terminal (N-term), central domain (central) and carboxy terminal (C-term)

\begin{tabular}{|c|c|c|c|c|c|c|c|c|}
\hline & \multicolumn{4}{|c|}{ Percent identity ${ }^{\mathrm{a}}$} & \multicolumn{4}{|c|}{ Percent similarity } \\
\hline & Overall & N-term & Central & C-term & Overall & $N$-term & Central & C-term \\
\hline lit/vir & 97.7 & 95.7 & 98.3 & 100.0 & 97.8 & 95.7 & 98.7 & 100.0 \\
\hline lit/mel & 77.8 & 55.1 & 90.7 & 92.4 & 83.7 & 66.0 & 93.7 & 95.5 \\
\hline vir/mel & 77.6 & 54.7 & 90.7 & 92.4 & 83.7 & 66.0 & 93.7 & 95.5 \\
\hline
\end{tabular}

aTo calculate the percent identity, amino acids opposite a gap in either species were ignored.

bPercent similarity is the sum of the percent identity and the percent of conservative substitutions between the listed species. Conservative substitutions are based upon the biochemical similarity of the amino acids: D/E, K/R/H, N/Q, S/T, I/L/V, F/W/Y and A/G (Smith and Smith, 1990).

conserved between species (Dong et al, 1993) accommodated two amino acid substitutions in RNP2 of the second RNA-binding motif between D. melanogaster and the two $D$. virilis group species. The site of the dissonance song mutation (site 548 in D. melanogaster; Rendahl et al, 1996) had Arg in all three species.

Changes in amino acid sequence can be the result of either natural selection or neutral evolution. The ratio of nonsynonymous (Ka) and synonymous (Ks) substitutions provides a measure of the strength of selective forces acting on amino acid level. High $\mathrm{Ka} / \mathrm{Ks}$ ratio $(>1)$ would indicate strong adaptive selection favouring nonsynonymous substitutions, leading to low constraint on protein sequence. As shown in Table $2, \mathrm{Ka} / \mathrm{Ks}$ ratios were low in each species comparison, suggesting that non $A$ amino acid sites are not targets of this kind of selection, but are rather under strong constraint, at least in the $D$. virilis group species.

In addition to amino acid substitutions, the species may differ by insertions and deletions. In particular, the repetitive areas of the developmental genes of Drosophila are often characterised by these events (Colot et al, 1988). As shown in Table 3, all the three species had stretches of repeated amino acid sequences in both terminals of the NONA. Most of the stretches found in exons 1 (Gly 1), 2 (Gly 2 and a shorter repeat) and 3 (Gly 3) consisted of perfect or imperfect glycine repeats. In addition, all species had imperfect QA repeats in exon 2 corresponding to the $\mathrm{N}$-terminal region. Most repeats were shorter in $D$. melanogaster than in the other two species. As is shown in Table 3, the lengths of the repeats also varied a lot within the species $D$. littoralis.

We calculated two measures for codon usage in non $A$ of the three species: the codon bias index (CBI; Bennetzen and Hall, 1982) and the effective number of codons (ENC; Wright, 1990). In a gene with extreme codon bias CBI will equal 1.0, while with random codon usage it will equal

Table 2 The ratio of nonsynonymous (Ka) to synonymous (Ks) substitutions per site in the nonA coding region between $D$. littoralis (lit), D. virilis (vir) and D. melanogaster (mel) overall and separately $5^{\prime}$, central and $3^{\prime}$ region

\begin{tabular}{lcccc}
\hline & Overall & 5' region & Central & 3' region \\
\hline $\mathrm{lit} / \mathrm{vir}$ & 0.064 & 0.086 & 0.052 & 0.029 \\
$\mathrm{lit} / \mathrm{mel}$ & 0.115 & 0.196 & 0.039 & 0.094 \\
vir/mel & 0.120 & 0.198 & 0.040 & 0.076 \\
\hline
\end{tabular}

Table 3 Repeat length variation in the coding region of non A gene in D. littoralis (lit) populations from Oulu, Kuopio and Savonlinna and the strain from Zurich, and the presence of the same repeats in D. virilis (vir) and D. melanogaster (mel)

\begin{tabular}{|c|c|c|c|c|}
\hline Species & Gly 1 & $G \ln -A l a^{\mathrm{a}}$ & Gly 2 & Gly 3 \\
\hline lit $(\mathrm{Ou})$ & $\begin{array}{l}\mathrm{G}_{9} \mathrm{SG}_{9} \\
\mathrm{G}_{19-20}\end{array}$ & $(\mathrm{QA})_{18}$ & $\mathrm{G}_{20-21}$ & $\mathrm{G}_{3} \mathrm{~N}_{2} \mathrm{G}_{12-13} \mathrm{VG}_{3} \mathrm{VG}$ \\
\hline lit $(\mathrm{Ku})$ & $\begin{array}{c}\mathrm{GVG}_{17} \\
\mathrm{GVG}_{7} \mathrm{SG}_{8}\end{array}$ & $(\mathrm{QA})_{18}$ & $\mathrm{G}_{20}$ & $\mathrm{G}_{3} \mathrm{~N}_{2} \mathrm{G}_{12-13} \mathrm{VG}_{3} \mathrm{VG}$ \\
\hline lit (Sa) & $\begin{array}{c}\mathrm{G}_{18-19} \\
\mathrm{G}_{9} \mathrm{SG}_{8-9} \\
\mathrm{G}_{18-19}\end{array}$ & $(\mathrm{QA})_{18}$ & $\mathrm{G}_{20-22}$ & $\mathrm{G}_{3} \mathrm{~N}_{2} \mathrm{G}_{9-13} \mathrm{VG}_{3} \mathrm{VG}$ \\
\hline $\begin{array}{l}\text { lit (Zurich) } \\
\text { vir (1431) } \\
\text { mel }\end{array}$ & $\begin{array}{c}\mathrm{G}_{9} \mathrm{SG}_{8} \\
\mathrm{G}_{11} \\
\mathrm{G}_{8} \mathrm{~b}\end{array}$ & $\begin{array}{l}(\mathrm{QA})_{20}{ }^{\mathrm{c}} \\
(\mathrm{QA})_{20}{ }^{\mathrm{c}} \\
(\mathrm{QN})_{9}{ }^{\mathrm{d}}\end{array}$ & $\begin{array}{l}\mathrm{G}_{20} \\
\mathrm{G}_{29} \\
\mathrm{G}_{10} \mathrm{~b}\end{array}$ & $\begin{array}{c}\mathrm{G}_{3} \mathrm{~N}_{2} \mathrm{G}_{12} \mathrm{VG}_{3} \mathrm{VG} \\
\mathrm{G}_{3} \mathrm{NG}_{12} \mathrm{VG} \\
\mathrm{G}_{6}\end{array}$ \\
\hline
\end{tabular}

amperfect repeat, where $\mathrm{Q}$ can be replaced by $\mathrm{H}$ and $\mathrm{A}$ can be replaced by L.

${ }^{b}$ Glycine repeat is interrupted by other amino acids.

Imperfect repeat, where $\mathrm{Q}$ can be replaced by $\mathrm{H}$ and $\mathrm{A}$ can be replaced by $\mathrm{L}$ or $\mathrm{N}$.

${ }^{\mathrm{d}}$ Imperfect repeat, where $\mathrm{Q}$ can be replaced by $\mathrm{H}$ and $\mathrm{N}$ can be replaced by $G, R$ or $V$.

Amino acid symbols: $A=$ Alanine, $\mathrm{G}=$ Glycine, $\mathrm{H}=$ Histidine, $\mathrm{L}=$ Leusine,$\quad \mathrm{N}=$ Asparagine, $\mathrm{Q}=$ Glutamine, $\mathrm{R}=$ Arginine, $\mathrm{V}=$ Valine; $\mathrm{S}=$ Serine.

0 . ENC (ranges from 20 to 61) is negatively correlated to the level of codon bias. McVean and Vieira (1999) have studied codon bias in 50 orthologous genes in $D$. virilis and D. melanogaster and found the average ENC in both species to be very similar (45.52 and 43.36, respectively). In the case of non A, all three Drosophila species had low CBI values ( 0.113 for $D$. littoralis, 0.112 for $D$. virilis and 0.140 for D. melanogaster), and higher than average ENC (50.7 for $D$. virilis and 52.5 for $D$. littoralis and $D$. melanogaster) indicating relatively random use of codons. In Gly repeats, GGT and GGC were the most frequently used Gly codons in all three Drosophila species, G-ending codons being almost absent in Gly repeat regions (except for one GGG codon in Gly1 in D. littoralis). Gly codon usage in repeat regions did not, however, differ significantly from that in the rest of the gene $\left(\chi^{2}\right.$-test, data not shown)

\section{Intraspecific nucleotide variation in nonA gene of $D$. littoralis}

We analysed altogether $\sim 1.3 \mathrm{~kb}$ from the non $A$ locus of six males from each of the three Finnish $D$. littoralis popu- 
lations $(\mathrm{Ou}, \mathrm{Ku}$ and $\mathrm{Sa})$. The regions examined included sequences from $5^{\prime}$ end (part of exons 1 and 2) and from 3 ' end (part of exons 3 and 4 and intron 3; see Figure 1). The D. littoralis samples had, altogether, 22 polymorphic sites including three amino acid variants in exon 1 at nucleotide positions 131 (Ser/Leu), 206 (Gly/Val) and 229 (Gly/Ser). Only one polymorphic site (A/T polymorphism) was present in intron 3 (see Figure 2) .

The imperfect Gln-Ala repeat (in exon 2) was invariable in length among all $D$. littoralis samples, while the lengths of the Gly repeats varied between individuals (Table 3). The length of a perfect stretch of glycines in exon 2 (Gly 2) varied between 20 and 22 amino acids. Variation in Gly repeats 1 and 3 was even larger, these repeats being often interrupted by Ser, Val or Asn. Eight out of $18(44 \%)$ of synonymous mutations were associated with the Gly regions representing about $19 \%$ of the analysed sites.

Possible divergence between the three D. littoralis samples from Oulu, Kuopio and Savonlinna was studied by calculating $F_{\text {st }}$ values for silent sites in $5^{\prime}$ and $3^{\prime}$ parts of the gene. The significance of the $F_{\text {st }}$ values was tested by permutation tests (Hudson et al, 1992). For the three populations $\mathrm{F}_{\mathrm{st}}$ for the $5^{\prime}$ end was $0.0576(P=0.1850$ for 1000 permutations) and for $3^{\prime}$ end $0.0000(P=0.4560)$. Consequently, the data for the three samples were combined to study nucleotide variation in Finnish $D$. littoralis population.

We calculated two measures of nucleotide diversity for $D$. littoralis data: $\pi$ is the observed average proportion of nucleotide differences between sequences (Nei, 1987) and $\theta$ is based on the number of segregating sites, being sensitive to existence of deleterious alleles at low frequency (Watterson, 1975). Insertion/deletion (indel) variation was not included in the estimates of nucleotide variability. Only the $5^{\prime}$ region had nonsynonymous mutations (three; Table 4). The average variability at synonymous sites in in this region was as small or even smaller than at the $3^{\prime}$ end, and variation in intron sites was surprisingly low. Tajima's D statistics (Tajima, 1989) was $-0.99020, P>0.10$ (two-tailed test, assuming beta distribution) for the coding region in $5^{\prime}$ region and $-0.5045, P>0.10$ for the coding region in $3^{\prime}$ region. Negative $D$ values suggest that the population size may recently have undergone drastic changes or that the samples included some deleterious alleles at low frequency. The nonsignificance of the $\mathrm{D}$ values shows, how-

Table 4 Nucleotide diversity in the non A gene of D. littoralis

\begin{tabular}{cccccc}
\hline & $\begin{array}{c}\text { N-term, } \\
\text { nsyn } \\
(577.04)\end{array}$ & $\begin{array}{c}\text { C-term, } \\
\text { nsyn } \\
(281.83)\end{array}$ & $\begin{array}{c}\text { N-term, } \\
\text { syn } \\
(184.96)\end{array}$ & $\begin{array}{c}\text { C-term, } \\
\text { syn } \\
(72.17)\end{array}$ & $\begin{array}{c}\text { C-term, } \\
\text { intron } \\
(79)\end{array}$ \\
\hline $\mathrm{S}$ & 3 & 0 & 12 & 6 & 1 \\
$\pi$ & 0.00112 & 0.0000 & 0.01392 & 0.02047 & 0.00662 \\
$\theta$ & 0.00151 & 0.0000 & 0.0 .886 & 0.02417 & 0.00368 \\
\hline
\end{tabular}

The number of nonsynonymous, synonymous and intron sites analysed for $\mathrm{N}$ - and C-terminals are shown in brackets. $S$ is the number of segregating sites, $\pi$ is the average number of differences per base pair and $\theta$ is Watterson's estimator based on the number of segregating sites (Watterson, 1975). Because of X-linkage, $\theta=3 \mathrm{~N}_{\mathrm{e}} \mu$, where $\mathrm{N}_{\mathrm{e}}$ is the effective population size and $\mu$ is the mutation rate per nucleotide site per generation.

ever, that the expectation of selective neutrality of mutations was not violated.

Recombination is known to affect the level of DNA polymorphism in Drosophila (Begun and Aquadro, 1992). Our non $A$ sequences showed evidence for a minimum of five recombination events in the history of the population samples, which shows that non $A$ gene, located close to proximal end of the $X$ chromosome in $D$. littoralis (Päällysaho et al, 2001), experiences recombination. All four possible gametic types were found in 21 out of 231 pairwise comparisons involving 22 polymorphic sites. Significant linkage equilibrium was detected only once (by $\chi^{2}$ method with sequential Bonferroni correction) in 36 pairwise comparisons involving nine informative polymorphic sites. The distance between the linked alleles was very short (6 bp). In our sample the recombination parameter $R$, which is based on the variance of the average number of nucleotide differences between pairs of sequences, was 0.0862. For an X-linked locus $\mathrm{R}=3 \mathrm{Nr}$ ( $\mathrm{N}$ is the population size and $\mathrm{r}$ is the recombination rate per sequence; Hudson, 1987).

The Swiss D. littoralis strain 1007, for which we sequenced the whole coding region but no introns, differed from the Finnish $D$. littoralis samples by 1 fixed synonymous substitution in a region sequenced for all strains. The average divergence level, D (Nei, 1987), was 0.0248 for synonymous sites and 0.0011 for nonsynonymous sites.

\section{Divergence between $D$. littoralis and $D$. virilis}

The combined data from $D$. littoralis samples were used for comparisons with a single non $A$ sequence from $D$. virilis. Instead of using here the $D$. virilis sequence by Campesan et al (2001), which reported only coding region sites, we sequenced the relevant $5^{\prime}$ and $3^{\prime}$ regions of non $A$ from $D$. virilis strain 1431. Sequences of the two $D$. virilis strains differed from each other in the coding region examined by one synonymous base substitution. The $D$. virilis 1431 sequence differed from $D$. littoralis sequences in 62 sites. Fifteen of these sites were located in intron 3. The average divergence level D (Nei, 1987) between the $D$. littoralis and $D$. virilis non A sequences was 0.1566 for synonymous sites, 0.0137 for nonsynonymous sites and 0.2271 for the introns, ie synonymous sites are diverging 10 times faster than replacement sites, but slower than intron sites. The standard error for the intron divergence level is, however, rather high as it is based on only 68 sites (79 sites in D. littoralis and 68 sites in D. virilis).

We applied the McDonald-Kreitman test (McDonald and Kreitman, 1991), which compares the number of sites that are polymorphic within the species to those fixed between the species for replacement vs. synonymous sites excluding gaps, to the $D$. littoralis $-D$. virilis data. Under neutrality, the ratios should be equal. There were a total of 17 synonymous polymorphic sites and three replacement polymorphic sites in $D$. littoralis sample, and 35 fixed synonymous subsititutions and 11 fixed replacement substitutions between $D$. littoralis and D. virilis (a region of $1312 \mathrm{bp}$ ). The ratios were nearly equal, consistent with the neutral model $(\mathrm{G}=0.696, \mathrm{P}=0.4041)$.

The ratio of polymorphisms to fixed differences across the non A sequence was studied with the DNA Slider program (McDonald, 1998). Heterogeneity was measured by the number of runs of polymorphic sites and fixed differences, where a run is a series of one or more sites of one 
kind preceded and followed by sites of the other kind. We used here two test statistics: the number of runs, which is most powerful for detecting patterns containing several peaks of polymorphism, and the KolmogorovSmirnov statistic, which is most powerful for detecting patterns in which one end of the gene has high polymorphism and the other end has low polymorphism (McDonald, 1996, 1998). Significance of these statistics was tested by performing repeated Monte Carlo simulations of a coalescent model using parameters estimated from silent site variation in the data and an arbitrary value for recombination (1000 simulations with five recombination parameters: $\mathrm{R}=2,4,6,8$ and 10). The data contained 29 runs showing no deviation from heterogeneity under a neutral model (the $P$ values with different recombination parameters varied from 0.5340 to 0.6225 ). The Kolmogorov-Smirnov statistic was 0.04584 . The $P$ values for this test with different recombination parameters were nonsignificant (0.3850 and 0.4420), which suggests that there was no single change across a gene from low to high polymorphism, ie the $5^{\prime}$ region did not show higher polymorphism than the $3^{\prime}$ end.

\section{Divergence between $D$. littoralis and $D$. melanogaster}

The $D$. melanogaster sequence differed from the $D$. littoralis sequences at 353 sites. The average divergence level between these two species was 0.6266 for synonymous and 0.2686 for nonsynonymous sites. These numbers may, however, not be quite accurate because of possible saturation in the number of fixed synonymous differences during the long diverge time between the species.

\section{Discussion}

Comparison of gene sequences between distantly related species has been a commonly used technique to identify biologically functional regions in genes. $D$. virilis and $D$. melanogaster, which have diverged 60 million years ago (Beverley and Wilson, 1984), have been a very popular species pair in such studies. For example, genes like engrailed, hunchback, master mind and neuralized (see Zhou and Boulianne, 1994) are highly ( $>80 \%$ amino acid identity) conserved between the two species. The present study showed very high $(98 \%)$ conservation in non $A$ amino acid sequence between two $D$. virilis group species, D. littoralis and D. virilis (diverged 20 Mya; Spicer, 1991). The central domain and C-terminus of the NONA were conserved (91-92\%) also between the two D. virilis group species and D. melanogaster, while the N-terminal regions showed divergence (only 55\% identity) between the species groups.

Comparison of gene sequences of distantly related species is not a very informative strategy when studying the reasons for gene divergence or conservation, because of the problems with sequence alignment and the possible saturation of sites for synonymous substitutions. One way to overcome these problems is to study sequence variation within the species and compare this variation to fixed differences between closely and distantly related species. We have used this strategy to find out whether the divergence of the $5^{\prime}$ sequence between $D$. melanogaster and $D$. virilis group species is due to lowered functional constraint, or whether it might be a consequence of positive selection affecting this region. non $A$ showed a high rate of synonymous substitutions and weak codon bias in both terminals in D. littoralis, which suggests that these regions are not affected by selection for speed and accuracy of translation (see Akashi, 1994). The average level of variability at synonymous sites was $1.40 \%$ in the $5^{\prime}$ region and $2.09 \%$ in the $3^{\prime}$ end, variation in both regions being higher than the average $(0.70 \%)$ found earlier for X-linked genes in D. virilis (Vieira and Charlesworth, 1999). In D. virilis, non $A$ is located at the proximal end of the $\mathrm{X}$ chromosome, bands $15 \mathrm{D} / 16 \mathrm{~A}$, and in D. littoralis it is even closer to the centromere (Päällysaho et al, 2001). Our results on nonA suggest that the finding of Vieira and Charlesworth (1999) of no reduction in variation and no evidence for suppression of recombination at the base of $D$. virilis $X$ chromosome is relevant also for $D$. littoralis. This is in contrast to $D$. melanogaster, where the base of all chromosome arms is a region of low recombination (Ashburner, 1989).

Comparison of non $A$ sequence polymorphism in $D$. littoralis with fixed differences between this species and $D$. virilis supports the neutral mutation hypothesis assuming purifying (negative) selection to be the most prevalent type of selection affecting the gene. Also, the fact that the ratio of polymorphisms to fixed differences was fairly uniform across the gene suggests that the gene is evolving neutrally in the $D$. virilis group species (McDonald, 1998).

Both the $5^{\prime}$ and $3^{\prime}$ regions of non $A$ gene had repeat regions showing high variability in length, but not in position, within and between the species. The presence of repeated sequences coding for amino acid stretches and their poor conservation between species are features shared with many developmental genes of Drosophila (see Colot et al, 1988). The repeat regions, and more generally the nucleotide sequences that comprise the variable regions of per protein, have been found to be hot spots for events that lead to DNA (and therefore protein) changes (Colot et al, 1988). The most obvious role of repeats could be at the level of protein secondary structure (Newfeld et al, 1993). Among the human proteins resembling NONA by their structure PSF and p54 ${ }^{\text {nrb }}$ are unusually rich in Gln and Pro in their N-termini (Dong et al, 1993). In addition, NONO has a repeat of $10 \mathrm{Gln}$ in the same place as the three Drosophila species have their last Gly repeat, preceding the central domain (Yang et al, 1993).

Rendahl et al (1996) have suggested that nonA has a role in mRNA processing in the central nervous system. However, all mutants of this gene that show a deficient song phenotype are also affected in vision (Stanewsky et al, 1996), and the possibility that nonA mutant phenotypes (like male song) are caused by nonspecific effects such as generalised effects of protein stability cannot be excluded (Rendahl et al, 1996). In the present study $D$. virilis, D. littoralis and D. melanogaster did not have any amino acid differences in RNP1 or at the site of dissonance mutation, which shows that species differences in male courtship song cannot be caused by point mutations at these sites. The regions, which varied most profoundly between $D$. littoralis males and between $D$. littoralis and D. virilis corresponded to the Gly repeats located in $\mathrm{N}$ and C-terminals in NONA. Whether these repeats could affect species-specificity of the male song characters, as Thr-Gly repeat in per gene has been found to do in $D$. melanogaster (Wheeler et al, 1991), remains to be studied. 


\section{Acknowledgements}

This work was supported by the Academy of Finland to SH and AH (projects 36166 and 44960). SC was supported by a grant from EC predoctoral training fellowship. We are grateful for CP Kyriacou and the two anonymous reviewers for constructive critics on the manuscript.

\section{References}

Akashi H (1994). Synonymous codon usage in Drosophila melanogaster: natural selection and translational accuracy. Genetics 136: 927-935.

Ashburner M (1989). Drosophila: A Laboratory Handbook. Cold Spring Harbor Laboratory Press: Cold Spring Harbor, New York.

Begun DJ, Aquadro CF (1992). Levels of naturally occurring DNA polymorphism correlate recombination rates in D. melanogaster. Nature 356: 519-520.

Bennetzen JL, Hall BD (1982). Codon selection in yeast. J Biol Chem 257: 3026-3031.

Besser HV, Schnabel P, Weiland C, Fritz E, Stanewsky R, Saumweber H (1990). The puff-specific Drosophila protein Bj6, encoded by the gene no-on-transient $A$, shows homology to RNA-binding proteins. Chromosoma 100: 37-47.

Beverley SH, Wilson AC (1984). Molecular evolution in Drosophila and higher Diptera. II. A time scale for fly evolution. J Mol Evol 21: 1-13.

Campesan S, Chalmers D, Sandrelli F, Megighian A, Peixoto AA, Costa $\mathrm{R}$ et al (2001). Comparative analysis of the nonA region in Drosophila identifies a highly diverged $5^{\prime}$ gene that may constrain nonA promoter evolution. Genetics 157: 751764.

Colot HV, Hall JC, Rosbash M (1988). Interspecific comparison of the period gene of Drosophila reveals large blocks of nonconserved coding DNA. EMBO J 7: 3929-3937.

Dong B, Horowitz DS, Kobayashi R, Krainer R (1993). Purification and cDNA cloning of HeLa cell p54 $4^{\text {nrb }}$, a nuclear protein with two RNA recognition motifs and extensive homology to human splicing factor PSF and Drosophila NONA/BJ6. Nucleic Acids Res 21: 4085-4092.

Glenn TC, Glenn SJ (1994). Rapid elution of DNA from agarose gels using polyester plug spin inserts (PEPSIs). TIG 10: 344.

Hotta Y, Benzer S (1970). Genetic dissection of the Drosophila nervous system by means of mosaics. Proc Natl Acad Sci USA 67: 1156-1163.

Hudson RR (1987). Estimating the recombination parameter of a finite population model without selection. Renet Res $\mathbf{5 0}$ 245-250.

Hudson RR, Boos DD, Kaplan NL (1992). A statistical test for detecting population subdivision. Mol Biol Evol 9: 138-151.

Jones KR, Rubin GM (1990). Molecular analysis of no-on-transient $A$, a gene required for normal vision in Drosophila. Neuron 4: 711-723.

Kenan DJ, Query CC, Keene JD (1991). RNA recognition: towards identifying determinants of specificity. Trends Biochem Sci 16: 214-220.

Kulkarni SJ, Steinlauf AF, Hall JC (1988). The dissonance mutant of courtship song in Drosophila melanogaster: isolation, behavior and cytogenetics. Genetics 118: 267-285.

McDonald JH (1996). Detecting non-neutral heterogeneity across a region of DNA sequence in the ratio of polymorphism to divergence. Mol Biol Evol 13: 253-260.
McDonald JH (1998). Improved test for heterogeneity across a region of DNA sequence in the ratio of polymorphism to divergence. Mol Biol Evol 15: 377-384.

McDonald JH, Kreitman M (1991). Adaptive protein evolution at the Adh locus in Drosophila. Nature 351: 652-654.

McVean GAT, Vieira J (1999). The evolution of codon preferences in Drosophila: A maximum-likelihood approach to parameter estimation and hypothesis testing. J Mol Evol 49: 63-75.

Nei M (1987). Molecular Evolutionary Genetics. Columbia University Press: New York.

Newfeld SJ, Schmid AT, Yedvobnick B (1993). Homopolymer length variation in the Drosophila gene mastermind. J Mol Evol 37: 483-495.

Patton JG, Porro EB, Galceran J, Tempst P, Nadal-Ginard B (1993). Cloning and characterization pf PSF, a novel premRNA splicing factor. Genes Dev 7: 393-406.

Päällysaho S, Huttunen S, Hoikkala A (2001). Identification of $X$ chromosomal restriction fragment length polymorphism markers and their use in a gene localisation study in Drosophila virilis and D. littoralis. Genome 44: 1-7.

Rendahl KG, Gaukhshteyn, N, Wheeler DA, Fry TA, Hall JC (1996). Defects in courtship and vision caused by amino acid substitutions in a putative RNA-binding protein encoded by the no-on-transient A (nonA) gene of Drosophila. J Neurosci 16: 1511-1522.

Smith RF, Smith TF (1990). Automatic generation of primary sequence patterns from sets of related protein sequences. Proc Natl Acad Sci USA 87: 118-122.

Spicer GS (1991). Molecular evolution and phylogeny of the Drosophila virilis species group as inferred by two-dimensional electrophoresis. J Mol Evol 33: 379-394.

Stanewsky R, Fry TA, Reim I, Saumweber H, Hall JC (1996). Bioassaying putative RNA-binding motifs in a protein encoded by a gene that influences courtship and visually mediated behavior in Drosophila: in vitro mutagenesis of non A. Genetics 143: 259-275.

Tajima F (1989). Statistical method for testing the neutral mutation hypothesis by DNA polymorphism. Genetics 123: 585-595.

Thompson JD, Gibson TJ, Plewniak F, Jeanmougin F, Higgins DG (1997). The ClustalX windows interface: flexible strategies for multiple sequence alignment aided by quality analysis tools. Nucleic Acids Res 24: 4876-4882.

Wheeler DA, Kyriacou CP, Greenacre ML, Yu Q, Rutila J, Rosbash M, Hall JC (1991). Molecular transfer of a speciesspecific courtship behaviour from Drosophila simulans to Drosophila melanogaster. Science 25: 1082-1085.

Vieira J, Charlesworth B (1999). X chromosome DNA variation in Drosophila virilis. Proc R Soc Lond B 266: 1905-1912.

Watterson GA (1975). On the number of segregating sites in genetical models without recombination. Theor Pop Biol 7, 256-275.

Wright $F$ (1990). The 'effective number of codons' used in a gene. Gene 87: 23-29.

Yamamoto D, Jallon J-M, Komatsu A (1997). Genetic dissection of sexual behavior in Drosophila melanogaster. Annu Rev Entomol 42: 551-585.

Yang Y-S, Hanke JH, Carayannopoulos L, Craft CM, Capra JD, Tucker PW (1993). NonO, a Non-POU-domain-containing, octamer-binding protein, is the mammalian homolog of Drosophila non ${ }^{\text {diss }}$. Mol Cell Biol 13: 5593-5602.

Zhou L, Boulianne GL (1994). Comparison of the neuralized genes of Drosophila virilis and D. melanogaster. Genome 37: 840-847. 